\title{
The combined impact of dependency on caregivers, disability, and coping strategy on quality of life after ischemic stroke
}

Sarah Dewilde ${ }^{1,2^{*}}$ (D) Lieven Annemans ${ }^{3}$, Andrew Lloyd ${ }^{4}$, Andre Peeters ${ }^{5}$, Dimitri Hemelsoet ${ }^{6}$, Yves Vandermeeren $^{7}$, Philippe Desfontaines ${ }^{8}$, Raf Brouns ${ }^{9,10}$, Geert Vanhooren ${ }^{11}$, Patrick Cras ${ }^{12}$, Boudewijn Michielsens ${ }^{13}$,

Patricia Redondo ${ }^{14}$ and Vincent Thijs ${ }^{15,16}$

\begin{abstract}
Background: To estimate the additional impact of coping and of being dependent on caregivers, over and above the large effects of disability on utility after ischemic stroke.

Methods: A total of 539 patients were recruited into an observational, retrospective study when returning for a check-up between 3 and 36 months after an ischemic stroke. Patients' modified Rankin Scale (mRS), dependency on caregivers, the Brandtstädter and Renner Coping questionnaire (with summary scores: Tenacity of Goal Pursuit (TGP) and Flexible Goal Adjustment (FGA) coping styles), EQ-5D-3 L and co-morbidities were evaluated.

Results: In multivariable regression, greater disability (mRS) resulted in large utility losses, between 0.06 for mRS 1 to 0.65 for mRS $5(p<0.0001)$. Dependency on caregivers caused an additional dis-utility of $0.104(p=0.0006)$ which varied by $\mathrm{mRS}(0.044,0.060,0.083,0.115,0.150$ and 0.173 for mRS $0-5)$. The effect of coping on utility varied by coping style, by the disability level of the patient and by his or her dependency on caregivers. FGA coping was associated with additional increases in utility $(p<0.0001)$ over and above the effect of disability and dependency, whereas TGA had no significant impact. FGA coping was associated with larger utility changes among more disabled patients (0.018 to 0.105 additional utility, for mRS 0 to mRS 5 respectively). Dependent patients had more to gain from FGA coping than patients who function independently of caregivers: utility gains were between 0.049 and 0.072 for moderate to high levels of FGA coping. In contrast, the same positive evolution in FGA coping resulted in 0.039 and 0.057 utility gain among independent patients. Finally, we found that important stroke risk factors and co-morbidities, such as diabetes and atrial fibrillation, were not predictors of EQ-5D utility in a multivariable setting.
\end{abstract}

Conclusions: This study suggests that treatment strategies targeting flexible coping styles and decreasing dependency on caregivers may lead to significant gains in quality of life above and beyond treatment strategies that solely target disability.

Keywords: EQ-5D, Stroke, Disability, Dependency on caregivers, Coping, PRO, Utilities

\footnotetext{
* Correspondence: sarahf.dewilde@ugent.be; sd@she-consulting.be

${ }^{1}$ Department of Public Health, Faculty of Medicine, University of Ghent,

Ghent, Belgium

${ }^{2}$ Services in Health Economics (SHE), Brussels, Belgium

Full list of author information is available at the end of the article
}

(c) The Author(s). 2019 Open Access This article is distributed under the terms of the Creative Commons Attribution 4.0 International License (http://creativecommons.org/licenses/by/4.0/), which permits unrestricted use, distribution, and reproduction in any medium, provided you give appropriate credit to the original author(s) and the source, provide a link to the Creative Commons license, and indicate if changes were made. The Creative Commons Public Domain Dedication waiver (http://creativecommons.org/publicdomain/zero/1.0/) applies to the data made available in this article, unless otherwise stated. 


\section{Introduction}

Stroke is the most common cause of acquired disability worldwide. In stroke survivors, quality of life (QoL) is variably affected by multiple factors $[1,2]$. These include patient characteristics (age, sex) [3, 4], stroke outcome (physical disability, repeat events) $[5,6]$, stroke-related complications (speech impediment, cognitive impairment, depression) $[3,7,8]$, psychological factors (problem-solving versus emotion-focused coping style) [9-13] and changed aspects of daily living (place of residence, dependency on caregivers, mobility, returning to leisure activities) [3, 14, 15]. Each of these factors are known to affect QoL, however no study has investigated the additional or combined effect of these factors.

The modified Rankin Scale (mRS) is the most frequently used global outcome scale in ischemic stroke [16]. The mRS measures the degree of impairment in bodily functions and structures [17, 18]. Although it is inclined towards motor function, it also takes into account patient autonomy and activities of daily living [19]. Several large studies have documented the variation in EuroQol-5D (EQ-5D) utilities for different levels of the mRS $[5,6,20]$, showing that disability was the major determinant of QoL after stroke. Dependency on caregivers, a frequent theme affecting QoL in stroke survivors, is closely linked to patients' place of residence, i.e. dependent patients are mostly living in inpatient facilities where help from caregivers is nearby [8, 21, 22]. The link between dependency and disability is evident, and whilst it is documented that dependency on caregivers also negatively affects QoL, it is unclear whether there is an additional effect on QoL over and above the effect of disability. Finally, the impact of coping style on patients' EQ-5D utility has also been documented. Coping is defined as the actions or emotions people develop to deal with stressful events. The literature on coping style demonstrates that stroke patients with flexible, problem-solving coping styles have higher QoL compared to patients with avoiding, resignation and denial coping styles, and have a lower probability of suffering from depression [9-13]. Coping strategies can be developed in a positive or negative way, and it has been shown that a good coping style can be learned [9, 23]. However, it is unclear what the specific impact of coping is on QoL in addition to dependency on caregivers and on the large and well-documented impact of disability.

This study set out to estimate the importance of a personal mind-set (coping ability), clinical factors (disability) and environmental factors (living in inpatient facilities) on stroke patients' quality of life. The aim was to investigate whether there is an additional effect on the utility value of coping and of being dependent on caregivers, over and above the well-known effect of disability. The purpose of investigating factors that affect quality of life of stroke patients is to identify treatment strategies in addition to physical therapy that can enhance the quality of these patients' lives.

\section{Methods \\ BOI study design}

In this observational, retrospective Burden of Illness (BOI) study, patients with ischemic stroke were recruited from ten hospitals in Belgium. Patient selection was on an "all comers" basis with stratification by mRS value and time since stroke (less versus more than 6 months). Patients were recruited when they returned to the hospital for a regular, scheduled in-person check-up visit after their index ischemic stroke. The timing of this visit varied between 3 to 36 months after stroke depending on the hospital's follow-up policy. Patients with a major disability who had an mRS value of 4 or 5 were less able to attend the outpatient clinic and were recruited and interviewed (with their caregivers) by telephone or at their place of residence in the last year of the study. The hospitals were distributed throughout Belgium and included teaching and regional hospitals. Data was collected between September 2010 and May 2013. The ethics committees of the individual participating hospitals approved the study and informed consent was obtained from all patients or their caregivers. The resource use and cost data from the BOI study were published elsewhere [24].

\section{Outcome measures}

At the time of patient's check-up visit to the clinic, the physicians completed an mRS assessment. Physicians also completed data on co-morbidities (diabetes, previous stroke, transient ischemic attack) and medical risk factors (hypertension, hypercholesterolemia, smoking, atrial fibrillation). QoL data were collected using the EQ-5D-3 L questionnaire, which is a generic instrument to measure people's health status and summarize patient's QoL based on five domains: mobility, self-care, usual activities, pain/discomfort, and anxiety/depression [25-27]. Information on these five domains was combined in a single index, called utility value (see details in the statistical section below); it is this utility value that is used as the main outcome variable in all analyses. Patients gave information on their place of residence and whether they needed any mobility aids (wheelchair, walker) at the time of the follow-up visit. The definition of dependency in this study is based on a combination of patients' place of residence, and whether or not patients need daily input from caregivers to perform their daily activities. Dependency on caregivers was therefore defined as living either in a nursing home, a rehabilitation home, or moving out of one's own home to live with a family member, or living at one's own home but 
with daily help from a caregiver. The patient was defined as being "independent" when he/she lived at his own home and did not need daily help from a caregiver. This definition is different from the "dependency" concept which is implicitly included in the mRS, where it is closely linked to mobility (unable to walk without assistance, being in a wheelchair, being bedridden) and the resulting need from assistance from others. The dependency concept in our study focused on patients who are dependent on the goodwill and time from caregivers to receive help, patients who may experience distress of having to move out of their own home in order to facilitate the assistance given, and the psychological weight of the burden they impose on family caregivers.

Coping was assessed using the assimilative-accommodative coping scale, consisting of two subscales: the assimilative coping or "tenacious goal pursuit" scale (TGP) which assesses whether patients can adjust the (new post-stroke) situation to their personal preferences; and the accommodative coping scale or "flexible goal adjustment" (FGA) which evaluates the opposite, namely whether patients can adjust their preferences according to the (new post-stroke) situation [28]. An example of the assimilative coping strategy would be to put more effort into occupational therapy session to be able to dress oneself again as this has been a personal goal ("When faced with obstacles, I usually double my efforts."). An example of accommodative coping would be to accept the fact that one will never be able to walk without a walker anymore despite the wish to be capable of walking without assistance ("After a serious drawback, I soon turn to new tasks"). Patients often use these two strategies simultaneously, and this might evolve over time with assimilative coping playing a greater role in the acute phase after stroke, and accommodative coping being more prevalent in the chronic phase.

\section{Statistical analysis}

The EQ-5D domain data were transformed into utility indexes using the published European algorithm [29]. The utility index takes a value between -0.0743 and 1.000 , with 0 representing death and 1 representing full health. This variable's distribution is usually left skewed, bound by 1 and presents floor- and ceiling effects, and it was transformed to make it more amenable to statistical modelling by taking the complement (=1-Utility Index) to make the distribution right skewed and positive. The $\mathrm{mRS}$ is a 7-point scale with scores ranging from 0 (no symptoms at all) to 6 (death); the statistical analysis considered its ordinal nature. Dependency is a dummy variable and takes values 0 (independent) or 1 (dependent). The coping subscales, TGP and FGA, were calculated as the sum score of 15 items each, ranging from 0 to 60 , with higher scores indicating a higher level of coping. These variables were treated as a continuous variable in regression analysis, or categorized into three mutuallyexclusive clusters "low", "medium" and "high" for use in figures.

The analysis was carried out in four steps; in first instance the univariable effects of time, the mRS, coping and dependency on utility are presented with boxplots. Secondly, it was investigated whether the coping strategies TGP and FGA were independent concepts from disability and dependency. Thirdly, a multivariable Generalized Linear Model (GLM) was estimated to disentangle and estimate the effects of disability, coping strategy and dependency on utility. A fully parameterized model was estimated including age, sex, co-morbidities, risk factors and socio-economic variables. Gradually, non-significant parameters were removed using the type 3 tests and adjusted Aikaike Information Criterion (AICC). The best fit to the data was a model with a normal distribution and log link, selected among the Identity and log link, Gamma and normal distributions. The selection was based on AICC as well as on the comparison of the range of the predicted utilities with the observed utilities. Testing for multicollinearity was performed with the variance inflation factor and with the Craemer's V [30, 31]. Finally, each domain of the EQ-5D was examined using a cumulative logit model estimating the likelihood of scoring lower on each domain of the EQ-5D. No adjustment was made for multiple testing. All analyses were conducted in SAS 9.4.

\section{Results}

\section{Step 1: Univariable effects on the EQ-5D utility of disability, dependency on caregivers, tenacity and flexibility}

A total of 539 patients who came for a scheduled follow-up visit after having an ischemic stroke were recruited in the BOI study dataset, patient characteristics are given in Table 1. Figures 1a-d depict boxplots showing the univariable relationship between the post-stroke EQ-5D utility, measured at a median of 6.1 months after stroke (IQR 3.7-14.8), and disability (mRS), dependency on caregivers, tenacity (TGP) and flexibility (FGA). Utility values differed significantly by the level of disability $(p<0.0001)$, with statistically significant differences found between each adjacent level of the mRS (all $p<$ 0.01) (Fig. 1a). When testing for variation of the mRS-related utilities by age and sex, it was observed that females had a smaller utility change due to disability than males $(p=0.0025)$, and utility values were also generally lower in females. No significant interaction with age was found.

To investigate the effect of dependency (Fig. 1b), we compared the utility value of patients needing daily help from caregivers $(N=221,41 \%)$ with patients who were able to live independently. The effect of dependency on caregivers on utility was to lower the post-stroke utility 
Table 1 Demographic and clinical characteristics

\begin{tabular}{llllllll}
\hline & $\mathrm{mRS} 0$ & $\mathrm{mRS} 1$ & $\mathrm{mRS} 2$ & $\mathrm{mRS} 3$ & $\mathrm{mRS} 4$ & $\mathrm{mRS} 5$ & All \\
\hline $\mathrm{N}$ & 125 & 116 & 111 & 93 & 73 & 21 & 539 \\
\% Female & $35.8 \%$ & $40.4 \%$ & $36.7 \%$ & $47.3 \%$ & $48.0 \%$ & $47.6 \%$ & $41.1 \%$ \\
Age (mean, SD) & $67.9(12.3)$ & $69.7(11.5)$ & $63.2(14.6)$ & $71.8(11.4)$ & $70.6(13.2)$ & $77.3(9.6)$ & $68.7(12.9)$ \\
Hypertension & $71.0 \%$ & $76.8 \%$ & $70.8 \%$ & $70.7 \%$ & $71.8 \%$ & $90.5 \%$ & $73.0 \%$ \\
Diabetes & $19.0 \%$ & $18.6 \%$ & $15.7 \%$ & $30.3 \%$ & $18.8 \%$ & $38.1 \%$ & $20.9 \%$ \\
Atrial fibrillation & $18.5 \%$ & $22.3 \%$ & $17.0 \%$ & $30.3 \%$ & $30.0 \%$ & $45.0 \%$ & $23.6 \%$ \\
Previous stroke & $15.6 \%$ & $6.5 \%$ & $13.6 \%$ & $17.4 \%$ & $9.7 \%$ & $33.3 \%$ & $13.3 \%$ \\
History of TIA & $19.4 \%$ & $4.8 \%$ & $3.4 \%$ & $10.9 \%$ & $12.9 \%$ & $22.2 \%$ & $10.4 \%$ \\
Months since diagnosis (mean, SD) & $11.2(11.5)$ & $12.5(17.1)$ & $10.2(10.1)$ & $18.8(25.5)$ & $19.6(72.1)$ & $11.8(12.1)$ & $13.8(30.8)$ \\
\hline
\end{tabular}

$\mathrm{SD}=$ standard deviation; $\mathrm{TIA}=$ Transient Ischemic Attack; $\mathrm{mRS}=$ modified Rankin Score

value in the BOI study by 0.377 , from 0.761 to 0.384 . The same effect was found when analysing these results by age and sex: no significant effect was found with higher age, but females had lower utility values regardless of dependency status, and also experienced a smaller utility decrement due to being dependent (0.358) than males $(0.405 ; p=0.020)$.

In Fig. 1c and $\mathrm{d}$ the relationship between utility and coping is shown. The coping variables were categorized into three levels, based on clustering analysis: TGP (low:

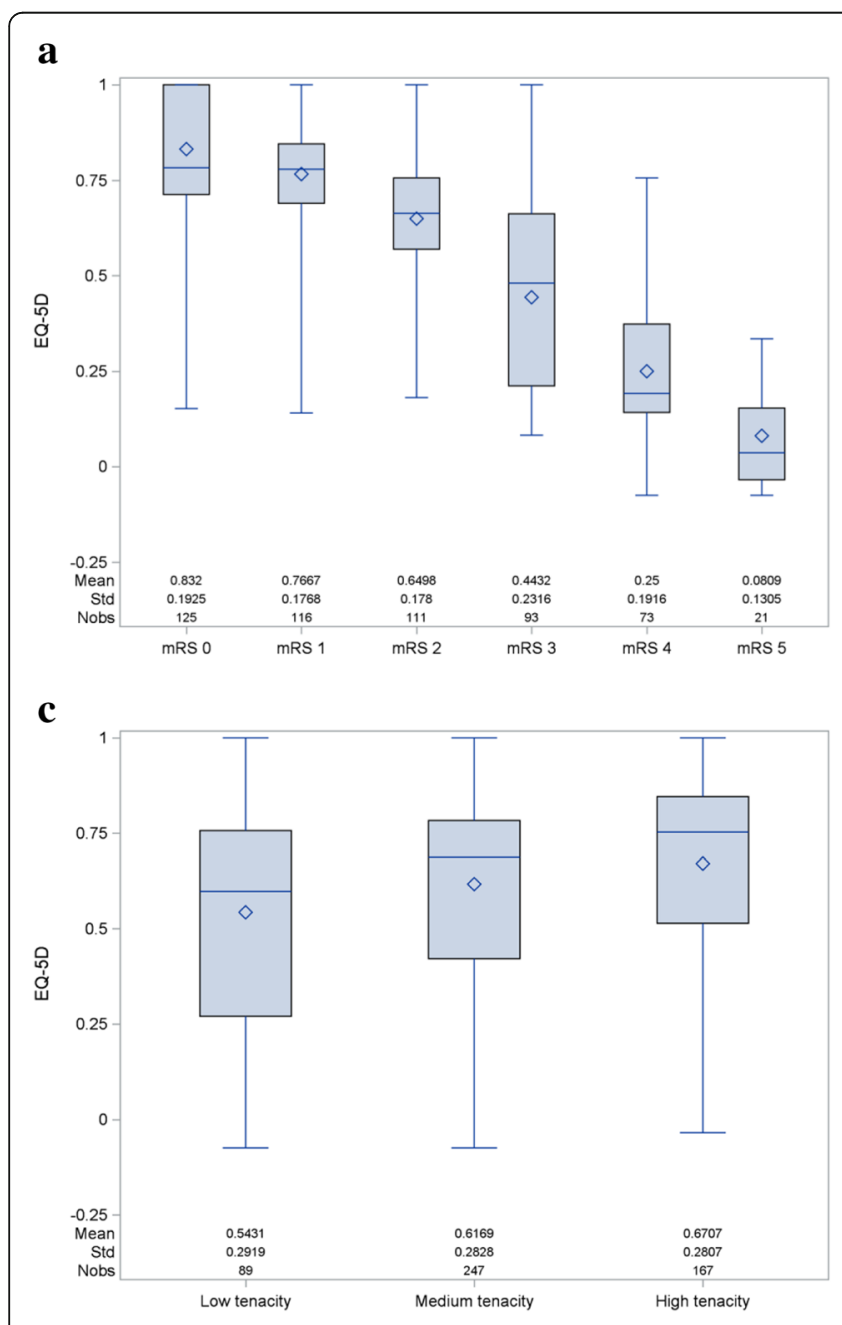

b

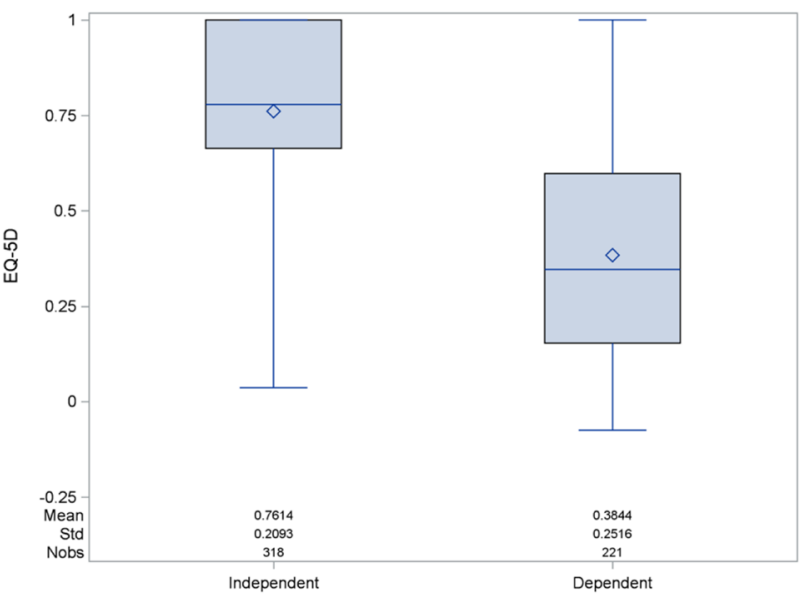

d

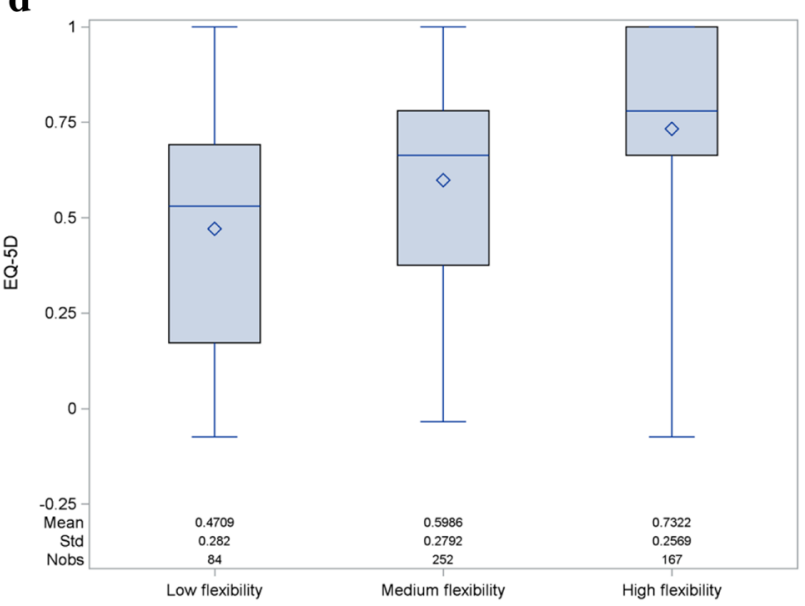

Fig. 1 Univariable relationship between the EQ-5D utility and mRS (1a), dependency on caregivers (1 b), tenacity (1c), and flexibility (1d) 
0-24, medium: 25-34, high: 35-60 score points) and FGA (low: 0-27, medium: 28-39, high: 40-60 score points). The average score points per TGP category were 19.54 (SD 4.65), 29.49 (SD 2.76) and 40.20 (SD 4.71), with an overall average of 31.28 (SD 8.21). The average score points per FGA category were 20.40 (SD 5.62), 34.13 (SD 3.22) and 44.49 (SD 3.62), with an overall average of 35.31 (SD 9.01). Patients with low levels of coping experienced lower EQ-5D utility values, and this can be observed for both the TGP and FGA strategies. The box plots show a stronger relationship between the EQ-5D utility and FGA compared to TGP. The utility increments for TGP were 0.0765 from low to medium tenacity and 0.0538 from medium to high tenacity. For FGA, the observed utility increases were 0.1277 for low to medium flexibility and 0.1336 for medium to high flexibility. Age and sex showed no significant effect (age: $p=0.079$ for TGP and $p=0.45$ for FGA, sex: $p=0.43$ for TGP and $p=0.94$ for FGA).
Step 2: The independence of the concepts of tenacity and flexibility

In Figs. 2a-d, the associations between coping (TGP and FGA) and the mRS and dependency on caregivers is displayed. No strong relationship is present with any of the coping concepts: the full range of TGP and FGA score points is present at any level of the mRS or dependency on caregivers. Furthermore, when examining the relationship between tenacity and flexibility, it is apparent that they measure two very different concepts: tenacity explains only $6 \%$ of the variation in flexibility (regression-based approach, R-square $=0.06$ ).

Step 3: Multivariable analysis: The relationships between disability, dependency on caregivers and coping, and their combined effect on utility

The concepts of disability, dependency on caregivers and coping (TGP, FGA) all have an influence on QoL, however their effect might not be additive. Two integrated models

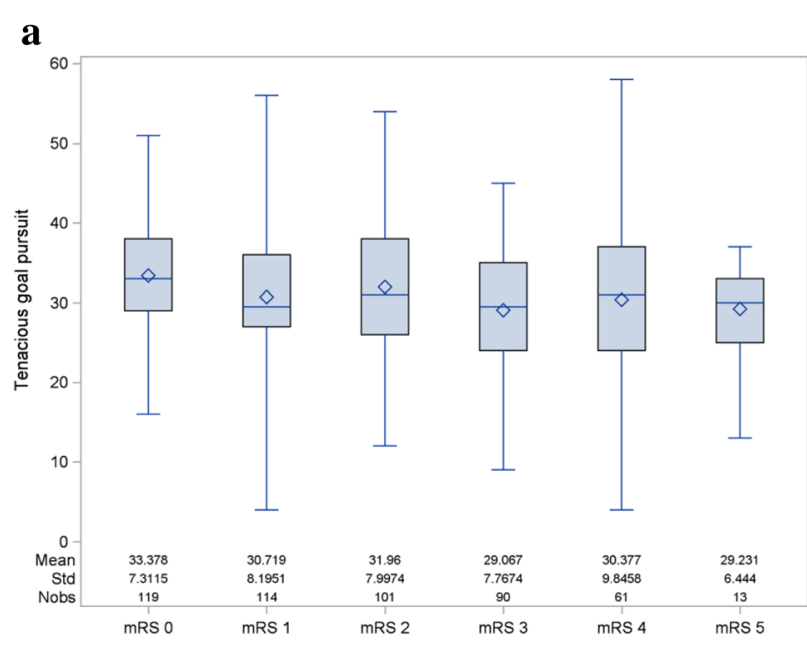

b

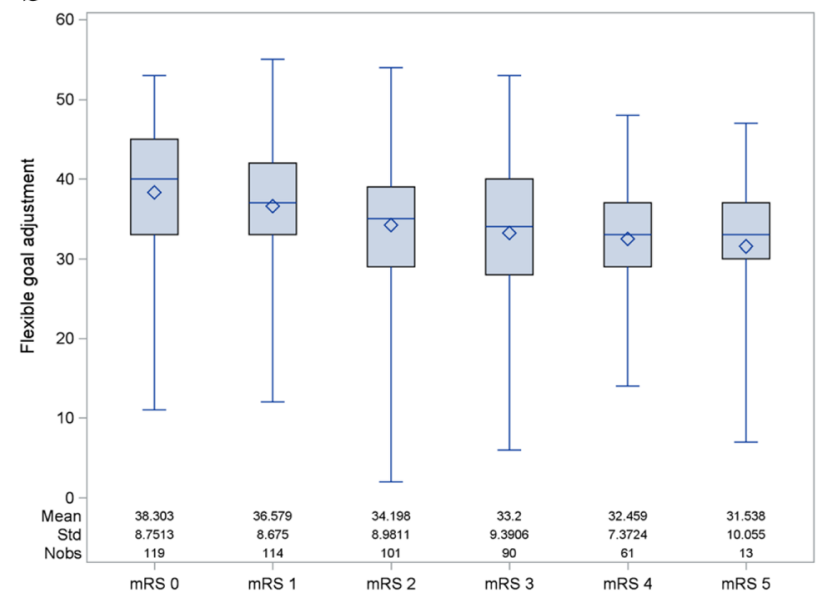

c

d
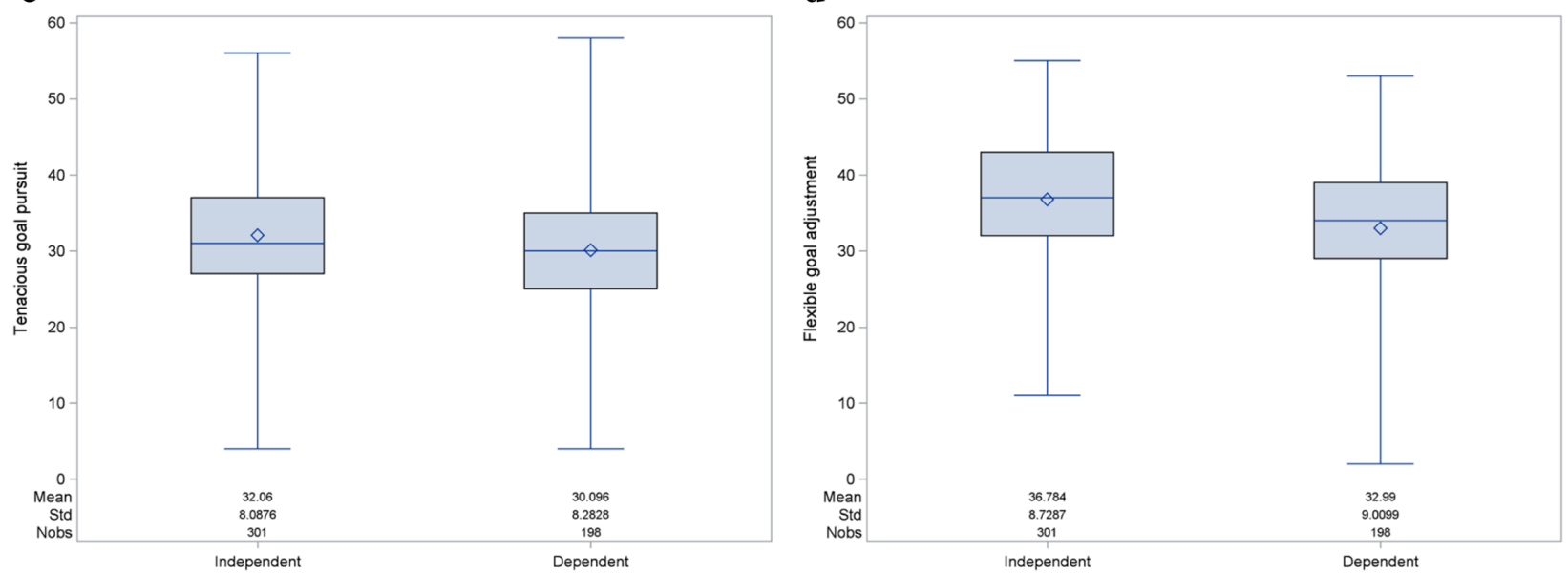

Fig. 2 Association between TGP and mRS (2a), FGA and mRS (2b), TGP and dependency on caregivers (2c) and FGA and dependency on caregivers (2d) 
were estimated: a simple model including only the mRS, dependency, TGP and FGA; and a more elaborate model adjusting these estimates for age and sex (Table 2). In this latter model, important risk factors (smoking, hypertension, hypercholesterolemia) and co-morbidities (diabetes, atrial fibrillation, previous stroke, TIA) associated with higher risk of stroke were explored but found not to be significant and therefore excluded. The mRS was the most significant contributor to explain the variance in utilities $(p<0.001)$, followed by FGA $(p<0.001)$ and dependency on caregivers $(p=0.0006)$. TGP was not significant in the simple model $(p=0.66)$, however a significant interaction was found with age in the elaborate model $(p=0.029)$. Age had the effect of increasing average utility values by 0.010 per 10 years of age $(p=0.017)$, ceteris paribus, whereas female sex reduced utility values by $0.028 \mathrm{com}-$ pared to males $(p=0.0368)$.

The utilities based on calculations from the simple model are displayed in Table 3: utilities were calculated for different combinations of mRS, dependency, TGP and FGA. Each time, the effect of one parameter was varied whilst keeping the other variables at average levels. Based on these utilities, utility decrements were derived for the effect of the mRS, dependency on caregivers, TGP and FGA levels; these values are presented in Table 4.

The mRS resulted in utility values varying between 0.813 and 0.263 ; the corresponding utility decrements were large: between -0.068 and -0.549 for mRS 1 to mRS 5 compared to mRS 0 . The additional effect of dependency on caregivers was significant both statistically and clinically: over and above the effect of disability

Table 2 Results of the multivariable regression

\begin{tabular}{|c|c|c|c|c|}
\hline & \multicolumn{2}{|c|}{ Simple model } & \multicolumn{2}{|c|}{ Elaborate model } \\
\hline & Parameter & $P$-value & Parameter & P-value \\
\hline Intercept & -1.4004 & & -0.3828 & \\
\hline mRS 1 & 0.3089 & $<.0001$ & 0.2890 & $<.0001$ \\
\hline $\mathrm{mRS} 2$ & 0.6403 & & 0.6357 & \\
\hline mRS 3 & 0.9596 & & 0.9529 & \\
\hline $\mathrm{mRS} 4$ & 1.2250 & & 1.2232 & \\
\hline mRS 5 & 1.3705 & & 1.3874 & \\
\hline $\begin{array}{l}\text { Dependency } \\
\text { on caregivers }\end{array}$ & 0.2298 & 0.0006 & 0.2180 & 0.0012 \\
\hline TGP & -0.0010 & 0.6642 & -0.0305 & 0.0234 \\
\hline FGA & -0.0096 & $<.0001$ & -0.0095 & $<.0001$ \\
\hline Age & & & -0.0146 & 0.017 \\
\hline Female & & & 0.0842 & 0.0368 \\
\hline Age*TGP & & & 0.0004 & 0.029 \\
\hline
\end{tabular}

(mRS), dependency further reduced a patient's utility after stroke on average by 0.104 . This additional utility reduction due to daily dependency on caregivers varied by $\mathrm{mRS}$ and was $-0.044,-0.060,-0.083,-0.115,-0.150$, 0.173 for mRS 0 to 5 , respectively. A gradient can be observed for the effect of being dependent on caregivers on utility values: this negative effect becomes larger as patients are more disabled, but even at moderate disability (e.g. $m R S=2)$, the effect of dependency $(-0.083)$ was found to be larger than the minimally important difference (MID) that is generally accepted for the EQ-5D utility values [32-35]. The effect of TGP on utility values was small in both models and below the threshold of the MID, as evidenced by the values in Table $4(-0002$ to -0.008$)$.

The effect of FGA was significant across its whole range and at all levels of the mRS and of patients' dependency status (Table 4). The potential utility increase from adopting a flexible coping style was largest among patients who are inflexible: coaching a patient from low $(\mathrm{FGA}=20.4)$ to medium flexibility $(\mathrm{FGA}=34.1)$ resulted in 0.063 utility gain, versus 0.043 utility gain from medium ( $\mathrm{FGA}=34.1)$ to high flexibility $(\mathrm{FGA}=44.6)$. Secondly, the effect of FGA was larger at higher mRS levels: the more disabled patients are, the more they can gain from a flexible attitude towards their goals. Estimated utility gains from learning a flexible coping style ranged from 0.018 for mRS 0 to 0.105 for mRS 5 (Table 4). Thirdly, it was found that the effect of FGA is also larger in patients who are dependent on caregivers compared to patients who are not. The utility increases among dependent patients resulting from low to medium, and medium to high flexibility were 0.072 and 0.049 , whereas the same improvement in flexible coping style among independent patients resulted in 0.057 and 0.039 utility gains (calculations based on Table 3).

\section{Step 4: The impact of disability, dependency and coping on the individual EQ-5D domains}

In Table 5 a set of odds ratios is presented for scoring one level lower (i.e. better) on each individual domain of the EQ-5D. Odds ratios lower than "1" indicate that the patient is more likely to experience problems in that domain with increasing values of that variable. Conversely, odds ratios higher than " 1 " indicate fewer problems with higher levels in this domain. Results show that patients with a higher mRS have more problems in each of the five domains, hence its strong impact on utility values. Furthermore, dependency on caregivers results in a higher likelihood of indicating problems with mobility, usual activities, and anxiety and depression. Surprisingly, no effect was found for dependency on problems with self-care. Likewise, patients with more flexible goal adjustment are likely to have fewer problems in most 
Table 3 Average utility values by mRS, dependency, tenacity and flexibility scores, calculations based on the simple model

\begin{tabular}{lc}
\hline Effect of mRS: & \\
Dependency: & 41\% Dependent \\
Tenacity: & Average: 31.3 \\
Flexibility: & Average: 35.3 \\
mRS 0 & 0.813 \\
mRS 1 & 0.745 \\
mRS 2 & 0.645 \\
mRS 3 & 0.511 \\
mRS 4 & 0.363 \\
mRS 5 & 0.263
\end{tabular}

Effect of Flexible Goal Adjustment:

\begin{tabular}{|c|c|c|c|c|c|c|}
\hline Dependency: & Dependent & Dependent & Dependent & Independent & Independent & Independent \\
\hline Tenacity: & Average: 31.3 & Average: 31.3 & Average: 31.3 & Average: 31.3 & Average: 31.3 & Average: 31.3 \\
\hline Flexibility: & Low: 20.4 & Med: 34.1 & High: 44.6 & Low: 20.4 & Med: 34.1 & High: 44.6 \\
\hline mRS 0 & 0.753 & 0.783 & 0.804 & 0.804 & 0.828 & 0.844 \\
\hline mRS 1 & 0.663 & 0.705 & 0.733 & 0.732 & 0.766 & 0.788 \\
\hline $\mathrm{mRS} 2$ & 0.531 & 0.589 & 0.628 & 0.627 & 0.673 & 0.705 \\
\hline $\mathrm{mRS} 3$ & 0.355 & 0.434 & 0.488 & 0.487 & 0.551 & 0.593 \\
\hline $\mathrm{mRS} 4$ & 0.159 & 0.263 & 0.333 & 0.331 & 0.414 & 0.47 \\
\hline mRS 5 & 0.027 & 0.147 & 0.229 & 0.227 & 0.322 & 0.387 \\
\hline
\end{tabular}

Effect of Tenacious Goal Pursuit:

\begin{tabular}{lllllll}
$\begin{array}{l}\text { Dependency: } \\
\text { Tenacity: }\end{array}$ & Dependent & Dependent & Dependent & Independent & Independent & Independent \\
Flexibility: & Average: 35.3 & Med: 29.5 & High: 40.2 & Low: 19.5 & Med: 29.5 & High: 40.2 \\
mRS 0 & 0.783 & Average: 35.3 & Average: 35.3 & Average: 35.3 & Average: 35.3 & Average: 35.3 \\
mRS 1 & 0.705 & 0.785 & 0.788 & 0.828 & 0.829 & 0.831 \\
mRS 2 & 0.589 & 0.708 & 0.711 & 0.765 & 0.768 & 0.77 \\
mRS 3 & 0.434 & 0.593 & 0.597 & 0.673 & 0.677 & 0.68 \\
mRS 4 & 0.262 & 0.44 & 0.446 & 0.55 & 0.555 & 0.56 \\
mRS 5 & 0.147 & 0.27 & 0.277 & 0.414 & 0.42 & 0.426 \\
\hline
\end{tabular}

Table 4 Utility differences due to dependency, flexibility and tenacity

\begin{tabular}{|c|c|c|c|c|c|c|}
\hline & $\mathrm{mRS}>0$ vs. $\mathrm{mRS}=0$ & $\begin{array}{l}\text { Dependent vs. } \\
\text { independent }\end{array}$ & $\begin{array}{l}\text { Low vs. medium } \\
\text { flexibility }\end{array}$ & $\begin{array}{l}\text { Medium vs. high } \\
\text { flexibility }\end{array}$ & $\begin{array}{l}\text { Low vs. medium } \\
\text { tenacity }\end{array}$ & $\begin{array}{l}\text { Medium vs. high } \\
\text { tenacity }\end{array}$ \\
\hline mRS 0 & ref & -0.044 & -0.027 & -0.018 & -0.002 & -0.002 \\
\hline mRS 1 & -0.0677 & -0.060 & -0.036 & -0.025 & -0.003 & -0.003 \\
\hline $\mathrm{mRS} 2$ & -0.1679 & -0.083 & -0.051 & -0.034 & -0.004 & -0.004 \\
\hline mRS 3 & -0.3015 & -0.115 & -0.070 & -0.047 & -0.005 & -0.005 \\
\hline $\mathrm{mRS} 4$ & -0.4500 & -0.150 & -0.091 & -0.062 & -0.006 & -0.007 \\
\hline mRS 5 & -0.5498 & -0.173 & -0.105 & -0.071 & -0.007 & -0.008 \\
\hline $\begin{array}{l}\text { Average } \\
\text { Effect }\end{array}$ & -0.307 & -0.104 & -0.063 & -0.043 & -0.004 & -0.005 \\
\hline Note & $\begin{array}{c}\text { With } 41 \% \text { patients } \\
\text { dependent, TGP = } 31.3 \\
\text { FGA }=35.3\end{array}$ & $\begin{aligned} \text { With TGP } & =31.3 \text { and } \\
F G A & =35.3\end{aligned}$ & $\begin{array}{l}\text { With } 41 \% \text { of patients } \\
\text { dependent and TGP }=31.3\end{array}$ & & \multicolumn{2}{|c|}{$\begin{array}{c}\text { With } 41 \% \text { of patients dependent } \\
\text { and } \mathrm{FGA}=35.3\end{array}$} \\
\hline
\end{tabular}


Table 5 Odds Ratios for scoring one level lower on each EQ-5D domain

\begin{tabular}{llllll}
\hline & Mobility & Self-care & Usual activities & Pain & Anxiety \& depression \\
\hline Age, per 10 additional years & $\mathbf{0 . 7 4 3}$ & 0.889 & $\mathbf{1 . 1 6 9}$ & $\mathbf{1 . 1 4 6}$ & 1.076 \\
Female & 0.772 & 1.074 & $\mathbf{0 . 5 5 5}$ & $\mathbf{0 . 6 3 9}$ & $\mathbf{0 . 5 1 0}$ \\
mRS & $\mathbf{0 . 3 1 6}$ & $\mathbf{0 . 1 7 5}$ & $\mathbf{0 . 2 6 4}$ & $\mathbf{0 . 6 5 1}$ & 0.906 \\
Dependent on others & $\mathbf{0 . 5 2 1}$ & 0.775 & $\mathbf{0 . 3 2 3}$ & 0.834 & $\mathbf{0 . 5 3 6}$ \\
Flexibility, per 10 additional point score & $\mathbf{1 . 3 5 9}$ & $\mathbf{1 . 4 2 9}$ & $\mathbf{1 . 5 9 0}$ & 1.029 & $\mathbf{2 . 2 7 7}$ \\
Tenacity, per 10 additional point score & 1.113 & 1.111 & 1.010 & 1.164 & 1.166 \\
\hline
\end{tabular}

Results in bold are statistically significant $p<0.05$

domains. Higher age was positively associated with mobility problems, and negatively associated with the likelihood of indicating problems with usual activities and pain. In addition, an association was found between female sex and the likelihood of problems with usual activities and pain. Finally, TGP was found not to have any impact on any domain of the EQ-5D.

\section{Discussion}

\section{Comparison of our findings with published literature}

The added value of our research is that it presents a multivariable analysis based on real-world data in which the effects of different determinants of QoL post-stroke are jointly estimated, and, moreover, that utility values are presented for use in economic evaluation. Our analysis demonstrated that dependency on caregivers plays an important role in influencing QoL, over and above the large effect of disability. Our study was able to quantify this additional effect (average reduction of 0.104 in utility) and showed a variation of the effect by level of physical disability. Furthermore, we found that coping style also has an important impact on utility, over and above the large effects of disability and dependency. The positive effect of coping varies by coping strategy (with FGA being associated with increases in utility, whereas TGA had no significant impact), by level of disability (higher disability being associated with higher utility increases due to FGA coping), and by dependency (dependent patients gain more utility from FGA coping than independent patients). We also found that important stroke risk factors and co-morbidities, such as diabetes and atrial fibrillation, were no significant predictors of QoL in a multivariable setting.

In contrast to our study, many previous publications have investigated the effect of determinants of utility in univariable analyses. Statistically significant utilities for each rank of mRS have been established based on large datasets $[5,6]$. The importance of dependency on caregivers was also previously highlighted: patients who depended on others for activities of daily living (ADL) were found to report a consistently lower QoL score up to two years after stroke, compared to independent patients [15]. A recent long-term European study showed that for dependency on caregivers for ADL the following factors were each individually correlated with EQ-VAS scores up to 5 years after stroke: the burden imposed on the caregiver, disability, depression and anxiety [7]. This study, however, did not present a multivariable analysis combining all these factors. A recent Dutch study [10] found associations between the Brandtstädter and Renner coping questionnaire and the WHO QoL-BREF assessment. Accommodative coping (FGA) was found to be associated with higher QoL in the chronic phase after stroke compared to assimilative coping (TGA). The authors also observed that FGA was neither associated with physical health (pain, sleep, energy, mobility, work, ADL), nor with social relationships or with environment (e.g. freedom, place of residence, financial resources, availability of care, transport). FGA however was associated with psychological health (feelings, cognition, self-esteem, beliefs). A recently published Dutch study [9] followed patients for one year after stroke and concluded that psychological factors (including coping style and depression), place of residence and independence in $\mathrm{ADL}$, were key in determining patients' evolution of QoL. This study did however not account for patient's disability and its large impact on QoL.

\section{Multi-disciplinary stroke care management}

It is of importance to gain a better understanding of the determinants of QoL after stroke, in order to target stroke care to what really matters to patients. Our findings show that patients with very different post-stroke health profiles could all benefit from care targeted towards (1) reducing disability, (2) decreasing dependency on caregivers, and (3) training in effective coping strategies in order to positively influence their QoL. While current strategies mostly target reduction of disability and increasing independence, less formal attention is given to coping strategies.

A few observational studies and one randomized trial examined the efficacy of coping treatment strategies in stroke patients. A Dutch study [36, 37] conducted a 1-year randomized controlled trial (RCT) among stroke patients to investigate the effect of Problem Solving Therapy (PST) on coping skills and on QoL. PST is a psychological 
therapy to help patients identify problems, determine goals, generate solutions, select the best option and assist in the evaluation of the result. This study found that PST, on top of physical rehabilitation and Occupational Therapy (OT), was positively associated with coping skills and with the EQ-5D utility. A cross-sectional study in 166 stroke patients in two rehabilitation centers [38] found that high depression scores were significantly related to less positive problem-solving and emotional coping, and a lower EQ-5D utility score. The authors concluded that all stroke patients could benefit from training in coping skills, especially problem-solving skills. Another publication [39] discusses the results of a 1-year prospective study on the effect of PST on coping skills and QoL of stroke patients. Findings were similar to the other studies: PST was found to be associated with better FGA and TGP coping, and patients with higher levels of FGA coping also had higher EQ-5D utility scores. TGP on the other hand was not found to have an effect on utility. Other publications discuss the efficacy of psychotherapy with PST in different patient populations [40, 41]. These studies all conclude that psychological therapy with a problem-solving orientation will lead to increased coping skills among patients. Our study demonstrated that increased coping skills, in particular FGA, is associated with higher QoL in stroke patients, regardless of their level of physical disability or whether they are dependent on caregivers.

Whilst all acute therapies aim to reduce long-term disability, occupational therapy to further increase independence has not been studied as extensively. A recent Cochrane Review [42] based on 9 studies and 994 patients determined that post-stroke occupational therapy increased extended ADL (including mobility, household tasks and leisure activities), improved functional capability and reduced poor outcomes (i.e. death, deterioration in $\mathrm{ADL}$, dependency in ADL or the need for institutional care). A few smaller clinical studies designed to investigate the efficacy of OT in gaining independence among stroke patients came to similar conclusions [43, 44]. Another systematic review [45] established that OT, with a focus on ADL-training, also improved basic ADL (dressing, feeding, hygiene) on top of extended ADL and the composite endpoint "poor outcome". These findings show that OT leads to increased independence in stroke patients, which is confirmed by our study showing that reduced dependency on caregivers is correlated with higher QoL.

The clinical implications of these findings are that OT, with a focus on gaining independence, and psychotherapy, geared towards an accommodative, problem-solving coping style, given in addition to physiotherapy are the most promising way for gaining additional units of QoL. Using the combined care of physiotherapists, occupational therapists and mental health support is expected to maximize the potential gains in QoL after stroke.

\section{Study limitations}

The main limitation of this research was the cross-sectional nature of the dataset: the study did not follow patients over time and was not able to capture the effect of changes in disability, dependency and coping on QoL. Furthermore, the wide range of time since stroke (between 3 and 36 months after stroke) may have influenced the results. A decrease in disease severity over time, due to higher mortality rates in more severe patients, is commonly observed in longitudinal studies (and was also found in the OxVASC study) [8]. This frequently results in a lower prevalence of severe patients in later time periods, causing an overestimation of utility values in later years after stroke. The BOI study was not biased due to the stratified design by $\mathrm{mRS}$ and by time since stroke. When we examined the patient characteristics over time, no different severity profile was found as time since stroke increased, and neither did the coping style. However, the different time-based samples may differ in unobserved characteristics that could affect outcome and QoL after stroke.

Furthermore, stroke may lead to major cognitive impairments and hence to the inability to participate in clinical studies. In the BOI study, patients were included in the study when they were coming back for a check-up; no exclusion criteria were defined based on patients' cognitive function. The patients' cognitive capabilities were not assessed, which is a limitation of our study. It is therefore not excluded that patients with cognitive problems responded themselves to our questions, possibly biasing results. In the BOI study, the caregiver (family member or nurse from the long-term facility) was allowed to assist their patient in filling in questionnaire. Seventy percent of patients filled in the EQ-5D themselves, whereas in 30\% of cases EQ-5D was filled in by a caregiver or nurse (with the help from the patient). Published literature shows that there is a reasonable correspondence between patient and proxy ratings for EQ-5D [46], and that differences between the ratings are smaller than the minimally important difference [47]. The difference in assessment between patient and proxy is found to be moderate for the EQ-5D-3 L instrument, and the concordance is generally better for the utility index than for the individual domain data $[48,49]$. We did not adjust our multivariable utility analysis for the type of EQ-5D responder (patient or caregiver) as this variable is strongly related to the mRS and to dependency on caregivers. Due to this correlation, we could not include this in the regression model without causing multicollinearity. $96 \%$ of the coping questionnaires were completed either by the patient or with explicit cooperation from the patient; in $4 \%$ of cases it was the caregiver who filled in the questionnaire without help from the patient. As the coping survey is very personal, informing about the patient's 
mind-set and how he/she deals psychologically with a difficult situation, it is likely that the answers given by the caregiver biased the results. At first sight, results showed that the lack of patient cooperation led to lower TGP (28.2 vs. 31.3) and FGA (29.9 vs. 35.5) scores for questionnaires filled in without patient input compared to the scores given by the patients themselves. This could suggest that caregivers underestimate the coping efforts of the patient. Taking into account the patient's age, gender and $\mathrm{mRS}$, this difference in coping scores by responder to the questionnaire became negligible and insignificant $(p=$ 0.49 for TGP and $p=0.09$ for FGA). We also investigated the effect of the lack of patient input on the coping questions on the EQ-5D values (controlling for mRS, age and gender) and found that it had no effect $(p=0.59)$. It cannot be excluded that the caregivers underestimated the patients' efforts in coping with their new post-stroke health state, but we did not consider this effect largely enough to exclude these data from our analysis.

The final limitation we would like to highlight, is the lack of a depression measurement in this study. The inclusion of depression scores, in relation to dependency and coping scores, would have been of added value for this multivariable analysis. It could have generated additional insights into the relative contribution of each factor on QoL after stroke, and it could have emphasised the role that psychotherapists may play in stroke rehabilitation.

\section{Conclusion}

In conclusion, QoL after stroke is determined by disability, the level of dependency on caregivers and patients' coping strategy. This study suggests that treatment strategies targeting flexible coping styles and decreasing dependency on caregivers may lead to significant gains in quality of life, above and beyond strategies that solely target disability. Therefore, a multi-disciplinary approach to stroke care, including physical rehabilitation for improving disability, occupational therapy for gaining independence in daily living and mental health support for training a flexible coping style and learning to apply this to all aspects of daily living is likely to result in highest QoL gains for stroke patients.

\footnotetext{
Abbreviations

ADL: Activities of daily life; AICC: Aikaike information criterion; BOI: Burden of Inness in stroke observational study; EQ-5D: EuroQoL-5 Dimensions; Exp: Exponent; FGA: Flexible goal adjustment; GLM: Generalized linear model; IQR: Inter-quartile range; Med: Medium; MID: Minimally important difference; mRS: Modified rankin scale; OR: Odds ratio; OT: Occupational therapy; PST: Problem solving therapy; QoL: Quality of life; RCT: Randomized controlled trial; SD: Standard deviation; TGP: Tenacious goal pursuit; TIA: Transient ischemic attack; WHO: World Health Organisation
}

\section{Funding}

The data collection was supported by the Belgian Stroke Council, a nonprofit association of neurologists promoting stroke prevention and improving care after stroke (http://belgianstrokecouncil.be). All co-authors from the Belgian Stroke Council contributed data towards this project.

\section{Availability of data and materials}

The dataset analysed during the current study is available from the corresponding author on reasonable request.

\section{Authors' contributions}

The members of the Belgian Stroke Council collected the data. Sarah Dewilde was responsible for the study design, data analysis and reporting of results, at the University of Ghent. Vincent Thijs, Lieven Annemans and Andrew Lloyd provided guidance on the research and reviewed the manuscript. All authors read and approved the final manuscript.

\section{Ethics approval and consent to participate}

The ethics committees of the individual participating hospitals approved the study and written informed consent was obtained from all patients or their caregivers.

\section{Consent for publication}

The manuscript has been read and approved by authors, all the authors are in agreement with the content of the manuscript and are aware of this submission.

\section{Competing interests}

The author declares that they have no competing interests.

\section{Publisher's Note}

Springer Nature remains neutral with regard to jurisdictional claims in published maps and institutional affiliations.

\section{Author details}

${ }^{1}$ Department of Public Health, Faculty of Medicine, University of Ghent, Ghent, Belgium. ${ }^{2}$ Services in Health Economics (SHE), Brussels, Belgium. ${ }^{3}$ Interuniversity Centre for Health Economics Research, University of Ghent, Vrije Universiteit Brussel, Ghent, Brussels, Belgium. ${ }^{4}$ Bladon Associates, Oxford, UK. ${ }^{5}$ Cliniques Universitaires Saint Luc, Brussels, Belgium. ${ }^{6}$ Ghent University Hospital, Ghent, Belgium. ${ }^{7}$ Université Catholique de Louvain, Yvoir, Belgium. ${ }^{8}$ Centre Hospitalier Chrétien, Liège, Belgium. 'Universitair Ziekenhuis Brussel, Brussels, Belgium. ${ }^{10}$ Center for Neurosciences (C4N), Vrije Universiteit Brussel (VUB), Brussels, Belgium. ${ }^{11}$ AZ Sint-Jan Brugge-Oostende AV, Bruges, Belgium. ${ }^{12}$ Born Bunge Institute, University and University Hospital, Antwerp, Belgium. ${ }^{13}$ Heilig Hart Ziekenhuis, Lier, Belgium. ${ }^{14} \mathrm{CHU}$ Ambroise Paré, Mons, Belgium. ${ }^{15}$ Stroke Division, Florey Institute of Neuroscience and Mental Health, University of Melbourne, Melbourne, Australia. ${ }^{16}$ Austin Health, Department of Neurology, Melbourne, Victoria, Australia.

Received: 7 July 2018 Accepted: 12 December 2018

Published online: 07 February 2019

\section{References}

1. Redon J, Olsen MH, Cooper RS, Zurriaga O, Martinez-Beneito MA, Laurent S, et al. Stroke mortality and trends from 1990 to 2006 in 39 countries from Europe and Central Asia: implications for control of high blood pressure. Eur Heart J. 2011;32(11):1424-31.

2. World Health Organization (WHO). The atlas of heart disease and stroke. http://www.who.int/cardiovascular_diseases/resources/atlas/en/index.html. Accessed October 2017.

3. Ayis S, Wellwood I, Rudd AG, McKevitt C, Parkin D, Wolfe CD. Variations in health-related quality of life (HRQOL) and survival 1 year after stroke: five European population-based registers. BMJ Open. 2015;5(6):e007101.

4. Bushnell CD, Reeves MJ, Zhao X, Pan W, Prvu-Bettger J, Zimmer L, et al. Sex differences in quality of life after ischemic stroke. Neurology. 2014;82(11): 922-31

5. Ali M, Maclsaac R, Quinn T. Dependency and health utilities in stroke: data to inform cost-effectiveness analyses. European Stroke Journal. 2017;2(1):70-6. 
6. Rivero-Arias O, Ouellet M, Gray A, Wolstenholme J, Rothwell PM, LuengoFernandez R. Mapping the modified Rankin scale (mRS) measurement into the generic EuroQol (EQ-5D) health outcome. Med Decis Mak. 2010;30(3):341-54.

7. De Wit L, Theuns P, Dejaeger E, Devos S, Gantenbein AR, Kerckhofs E, et al. Long-term impact of stroke on patients' health-related quality of life. Disabil Rehabil. 2017:39(14):1435-40.

8. Moeller D, Carpenter C. Factors affecting quality of life for people who have experienced a stroke. Int J Ther Rehabil. 2013;20(4):207-16.

9. van Mierlo M, van Heugten C, Post MWM, Hoekstra T, Visser-Meily A. Trajectories of health-related quality of life after stroke: results from a oneyear prospective cohort study. Disabil Rehabil. 2018;40(9):997-1006.

10. Visser MM, Aben L, Heijenbrok-Kal MH, Busschbach JJ, Ribbers GM. The relative effect of coping strategy and depression on health-related quality of life in patients in the chronic phase after stroke. J Rehabil Med. 2014;46(6):514-9.

11. Nijsse B, van Heugten CM, van Mierlo ML, Post MW, de Kort PL, Visser-Meily JM. Psychological factors are associated with subjective cognitive complaints 2 months post-stroke. Neuropsychol Rehabil. 2017;27(1):99-115.

12. van Rijsbergen MWA, Mark RE, Kop WJ, de Kort PLM, Sitskoorn MM. Psychological factors and subjective cognitive complaints after stroke: beyond depression and anxiety. Neuropsychol Rehabil. 2018:1-14.

13. Arwert HJ, Meesters JJL, Boiten J, Balk F, Wolterbeek R, Vliet Vlieland TPM. Post stroke depression: a long term problem for stroke survivors. American journal of physical medicine \& rehabilitation. 2018;97(8):565-71.

14. O'Sullivan C, Chard G. An exploration of participation in leisure activities post-stroke. Aust Occup Ther J. 2010;57(3):159-66.

15. van Mierlo ML, van Heugten CM, Post MW, Hajos TR, Kappelle $\sqcup$, VisserMeily JM. Quality of life during the first two years Post stroke: the Restore4Stroke cohort study. Cerebrovasc Dis. 2016;41(1-2):19-26.

16. Lees KR, Bath PM, Schellinger PD, Kerr DM, Fulton R, Hacke W, et al. Contemporary outcome measures in acute stroke research: choice of primary outcome measure. Stroke. 2012;43(4):1163-70.

17. Rankin J. Cerebral vascular accidents in patients over the age of 60. II. Prognosis. Scott Med J. 1957;2(5):200-15.

18. van Swieten JC, Koudstaal PJ, Visser MC, Schouten HJ, van Gijn J. Interobserver agreement for the assessment of handicap in stroke patients. Stroke. 1988;19(5):604-7.

19. Adams HP Jr, Chollet F, Thijs V. Measuring autonomy and functional recovery after stroke: implications for clinical trials testing medications during rehabilitation. J Stroke Cerebrovasc Dis. 2015;24(11):2429-33.

20. Luengo-Fernandez R, Gray AM, Bull L, Welch S, Cuthbertson F, Rothwell PM. Quality of life after TIA and stroke: ten-year results of the Oxford vascular study. Neurology. 2013;81(18):1588-95.

21. Cruice M, Hill R, Worrall L, Hickson L. Conceptualising quality of life for older people with aphasia. Aphasiology. 2010;24(3):327-47.

22. Tariah HA, Hersch G, Ostwald SK. Factors associated with quality of life: perspectives of stroke survivors. Physical \& Occupational Therapy In Geriatrics. 2006;25(2):33-50

23. Donnellan C, Hevey D, Hickey A, O'Neill D. Defining and quantifying coping strategies after stroke: a review. J Neurol Neurosurg Psychiatry. 2006;77(11):1208-18.

24. Dewilde $S$, Annemans $L$, Peeters A, Hemelsoet $D$, Vandermeeren $Y$, Desfontaines $P$, et al. Modified Rankin scale as a determinant of direct medical costs after stroke. Int J Stroke. 2017;12(4):392-400.

25. EuroQol. A new facility for the measurement of health-related quality of life. Health Policy. 1990;16(3):199-208.

26. Brooks R. EuroQol: the current state of play. Health Policy. 1996;37(1):53-72.

27. Van Reenen M, Oppe M. EQ-5D-3L User Guide. Basic information on how to use the EQ-5D-3L instrument. EuroQol Research Foundation. Version 5.1. April 2015. https://euroqol.org/wp-content/uploads/2016/09/EQ-5D-3L_ UserGuide_2015.pdf. Accessed October 2017.

28. Brandtstadter J, Renner G. Tenacious goal pursuit and flexible goal adjustment: explication and age-related analysis of assimilative and accommodative strategies of coping. Psychol Aging. 1990;5(1):58-67.

29. Greiner W, Weijnen T, Nieuwenhuizen M, Oppe S, Badia X, Busschbach J, et al. A single European currency for EQ-5D health states. Results from a sixcountry study. Eur J Health Econ. 2003;4(3):222-31.

30. Belsley DA. Conditioning diagnostics: collinearity and weak data in regression. New York: J. Wiley; 1991.

31. O'Brien RM. A caution regarding rules of thumb for variance inflation factors. Qual Quant. 2007:41(5):673-90.
32. Barton GR, Sach TH, Avery AJ, Jenkinson C, Doherty M, Whynes DK, et al. A comparison of the performance of the EQ-5D and SF-6D for individuals aged $>$ or $=45$ years. Health Econ. 2008;17(7):815-32.

33. Sullivan PW, Lawrence WF, Ghushchyan V. A national catalog of preferencebased scores for chronic conditions in the United States. Med Care. 2005; 43(7):736-49.

34. Marra CA, Woolcott JC, Kopec JA, Shojania K, Offer R, Brazier JE, et al. A comparison of generic, indirect utility measures (the HUI2, HUI3, SF-6D, and the EQ-5D) and disease-specific instruments (the RAQoL and the HAQ) in rheumatoid arthritis. Soc Sci Med. 2005;60(7):1571-82.

35. Walters SJ, Brazier JE. Comparison of the minimally important difference for two health state utility measures: EQ-5D and SF-6D. Qual Life Res. 2005; 14(6):1523-32.

36. Visser MM, Heijenbrok-Kal MH, van't Spijker A, Ribbers GM, Busschbach JJ. The effectiveness of problem solving therapy for stroke patients: study protocol for a pragmatic randomized controlled trial. BMC Neurol. 2013;13:67.

37. Visser MM, Heijenbrok-Kal MH, Van't Spijker A, Lannoo E, Busschbach JJ, Ribbers GM. Problem-solving therapy during outpatient stroke rehabilitation improves coping and health-related quality of life: randomized controlled trial. Stroke. 2016:47(1):135-42.

38. Visser MM, Heijenbrok-Kal MH, Spijker AV, Oostra KM, Busschbach JJ, Ribbers GM. Coping, problem solving, depression, and health-related quality of life in patients receiving outpatient stroke rehabilitation. Arch Phys Med Rehabil. 2015:96(8):1492-8.

39. Darlington AS, Dippel DW, Ribbers GM, van Balen R, Passchier J, Busschbach J. A prospective study on coping strategies and quality of life in patients after stroke, assessing prognostic relationships and estimates of costeffectiveness. J Rehabil Med. 2009;41(4):237-41.

40. Doorenbos A, Given B, Given C, Verbitsky N, Cimprich B, McCorkle R. Reducing symptom limitations: a cognitive behavioral intervention randomized trial. Psycho-Oncology. 2005;14(7):574-84.

41. van den Hout JH, Vlaeyen JW, Heuts PH, Zijlema JH, Wijnen JA. Secondary prevention of work-related disability in nonspecific low back pain: does problemsolving therapy help? A randomized clinical trial. Clin J Pain. 2003;19(2):87-96.

42. Legg LA, Lewis SR, Schofield-Robinson OJ, Drummond A, Langhorne P. Occupational therapy for adults with problems in activities of daily living after stroke. The Cochrane database of systematic reviews. 2017;7:Cd003585.

43. Petruseviciene D, Krisciunas A. Evaluation of activity and effectiveness of occupational therapy in stroke patients at the early stage of rehabilitation. Medicina (Kaunas). 2008;44(3):216-24.

44. Almhdawi KA, Mathiowetz VG, White M, RC dM. Efficacy of occupational therapy task-oriented approach in upper extremity Post-stroke rehabilitation Occup Ther Int. 2016;23(4):444-56.

45. IPB R. Occupational Therapy Part III. Occupational therapy for patients after stroke. In: HTA- Projektbericht 59; 2012. http://eprints.hta.lbg.ac.at/973/\#. Accessed: October 2018

46. Gabbe BJ, Lyons RA, Sutherland AM, Hart MJ, Cameron PA. Level of agreement between patient and proxy responses to the EQ-5D health questionnaire 12 months after injury. The journal of trauma and acute care surgery. 2012;72(4):1102-5.

47. Dinglas VD, Gifford JM, Husain N, Colantuoni E, Needham DM. Quality of life before intensive care using EQ-5D: patient versus proxy responses. Crit Care Med. 2013:41(1):9-14.

48. Davis JC, Hsiung GY, Bryan S, Jacova C, Jacova P, Munkacsy M, et al. Agreement between patient and proxy assessments of quality of life among older adults with vascular cognitive impairment using the EQ-5D-3L and ICECAP-O. PLoS One. 2016:11(4):e0153878.

49. Devine A, Taylor SJ, Spencer A, Diaz-Ordaz K, Eldridge S, Underwood M. The agreement between proxy and self-completed EQ-5D for care home residents was better for index scores than individual domains. J Clin Epidemiol. 2014:67(9):1035-43. 